\title{
Familles recomposées et ancrage résidentiel 1
}

\section{Céline Clément \\ Catherine Bonvalet}

Depuis les années 1970, de nombreuses transformations familiales et conjugales sont observées. On assiste notamment à une augmentation rapide du nombre de divorces, et à la formation de «nouvelles » configurations familiales, comme les ménages monoparentaux et les familles recomposées, ainsi qu'à la succession de plusieurs « séquences familiales » au long du cycle de vie, remettant en cause l'image d'un modèle familial homogène, stable et durable, et par là même, la notion de la famille, limitée au cadre du ménage. Si les familles recomposées ont toujours existé, comme en témoignent les différentes études historiques (Burguière, 1993 ; Flandrin, 1984), les modalités de leur formation se sont modifiées : elles sont davantage liées aujourd'hui à un divorce qu'à un décès de l'un des parents, offrant la possibilité aux enfants de maintenir le lien avec leurs deux parents. Avec les

Céline Clément, docteure en sociologie et démographie, INED - Cerpos.

clement@ined.fr

Catherine Bonvalet, directeur de recherche, co-responsable de l'unité Mobilité, territoires, habitat et sociabilité à l'INED.

Bonvalet@ined.fr

1. Recherche soutenue par le PUCA dans le cadre du programme « habitat et vie urbaine ». 
familles recomposées liées à un divorce, de nouvelles interrogations sont apparues, notamment sur la place du père, de la mère, du beau-père et de la belle-mère qui se doivent de composer un rôle « inédit » (Théry, 1987; Le Gall et Martin, 1993 ; Blöss, 1996, 1997 ; Cadolle, 2000 ; Martial, 2003), mais aussi sur la définition même de la famille - qui « dépasse les murs de la maison ». Sociologues et démographes ont alors été conduits à réexaminer la notion de famille en dépassant le cadre strict de ménage et donc de la corésidence (Desplanques, 1993 ; Bonvalet et Lelièvre, 1995 ; Bonvalet, 1997 ; de Singly, 1997). Ainsi, la définition de la famille, qui ne retient que les individus apparentés vivant dans le même logement, n'apparaît plus pertinente : le territoire des enfants des familles recomposées se « dualise », se multiplie. Outre les limites liées à la définition « classique » de la famille, les recherches sur les familles recomposées ont aussi mis en évidence les limites d'une vision trop statique des réalités familiales, et ont souligné l'importance d'intégrer une dimension temporelle à l'analyse, en s'attachant notamment à décrire les différentes séquences de vie familiale ainsi que leur enchaînement. En effet, les structures familiales observées à un moment donné sont le résultat d'un processus. Seule une analyse longitudinale permet alors d'apprécier «l'empreinte des trajectoires biographiques »(Blöss, 1996). Finalement, sociologues et démographes proposeront d'élargir le concept statistique de famille au-delà de la notion de résidence et de l'étendre à l'ensemble du réseau familial issu de l'histoire conjugale des parents. Dès lors, les familles recomposées sont aujourd'hui définies comme des constellations familiales, dessinées par l'espace de circulation des enfants entre les foyers paternels et maternels, où les dimensions de temps et d'espace apparaissent fondamentales (Théry, Blöss, Cadolle, Le Gall et Martin, Martial, op. cit.).

Néanmoins, ces études demeurent essentiellement centrées sur la résidence principale des parents ou des beaux-parents. Or, ce niveau d'analyse est encore insuffisant : comme l'ont souligné plusieurs recherches sur la famille et le logement, il est nécessaire de dépasser la vision d'une habitation unique, d'intégrer la multiplication des lieux d'habitat, et de tenir compte des durées longues de l'existence (Bonnin et de Villanova, 1999). Isabelle Bertaux Wiame (1995) a ainsi mis en évidence la nécessité de prendre en considération le contexte familial élargi. Un phénomène de logement multiple est à l'œuvre, et ce, dans toutes les catégories sociales, où la résidence dite « secondaire » apparaît «fortement investie sur les plans matériel, social et symbolique ». L'espace habité ne se résume donc pas au logement principal et "peut prendre la forme d'un véritable archipel résidentiel» (Bonnin et de Villanova, $o p$. cit.). En outre, s'il a été montré combien la seconde résidence pouvait être un support d'une identité familiale, il semble ici nécessaire de vérifier cette symbolique auprès des familles recomposées. Dans cette perspective, l'achat d'une seconde résidence, mais aussi l'investissement dans un lieu d'ancrage 
(la résidence principale des grands-parents, d'une tante, de lieux de vacances) peuvent être également des indicateurs de ce fonctionnement familial où l'on cherche à construire, à instaurer et à maintenir des liens entre enfants, beauxenfants, entre frères et sœurs, demi-frères et demi-sœurs, quasi frères et quasi sœurs, mais aussi entre petits-enfants. C'est pourquoi les notions d'espaces de vie (Courgeau, 1975 ; Frémont, 1984), de système résidentiel (Pinson, 1988 ; Barbary et Dureau, 1993) comme phénomènes de pluri-résidences, de circulations et de liens, sont proposées dans cet article. Ces concepts - qui dépassent le prisme de la résidence habituelle et intègrent l'ensemble des lieux fréquentés - permettent ainsi de suivre au cours du temps et dans l'espace non plus une seule localisation, mais plusieurs, qui peuvent évoluer au cours des parcours individuels. Aussi, si la corésidence et le « vécu partagé de l'enfance » apparaissent comme le socle des relations entre parents, enfants, beaux-parents, beaux-enfants, plus encore entre frères, sœurs, demi-frères et demi-sœurs (Martial, 2003 ; Poittevin, 2003), on s'intéressera ici aux divers lieux fréquentés - passés et présents - par les membres d'une recomposition familiale et les valeurs qui y sont attachées à travers les relations familiales impliquées autour de ces lieux.

\section{Présentation de la recherche}

\section{Présentation de l'enquête «Biographies et entourage»}

Cette recherche est constituée par une enquête qualitative auprès de personnes ayant recomposé une famille. Les entretiens ont été réalisés à partir de l'enquête par questionnaire «Biographies et entourage »..$^{2}$ de l'INED. Cette enquête, qui retrace la vie professionnelle, résidentielle et familiale des individus porte sur des générations nées entre 1930 et $1950^{3}$. Elle explore l'en-

2. Cette enquête a reçu le soutien financier de la CNAF - Caisse nationale d'allocations familiales, la CNAV - Caisse nationale d'assurance vieillesse, la DPM - Direction de la population et des migrations, la DREES - Direction de la recherche, des études, de l'évaluation et des statistiques, la DREIF - Direction régionale de l'équipement d'Île-de-France, l'IAURIF - Institut d'aménagement et d'urbanisme de la région Île-de-France, l'ODEP Mairie de Paris Observatoire du développement économique parisien, la RATP - Régie autonome des transports parisiens et le ministère de la Recherche, Action concertée incitative ville. Elle s'inscrit dans la continuité des enquêtes de l'INED : l'enquête « Triple biographie » (1981), « Peuplement et dépeuplement de Paris » (1986), «Proches et parents ». Elle prolonge notamment le concept d'entourage (Bonvalet et Lelièvre, 1995 ; Bonvalet et Maison, 1999).

3. La collecte, achevée en juillet 2001, comprend 2830 questionnaires en Île-de-France, dont un échantillon représentatif de 585 interviews à Paris intra-muros. Chaque questionnaire fournit systématiquement, en plus des descriptions détaillées concernant un univers varié de personnes et de lieux, les trajectoires familiales professionnelles et résidentielles de l'enquêté(e), de ses parents, ses conjoints soit 11 à 12000 biographies interconnectées. 
tourage des individus : les membres de leur lignée (père, mère, enfants, petits-enfants), de leurs collatéraux (frères, sœurs, demi-sœurs, demi-frères et sœurs), de la conjugalité (conjoints, parents et enfants de ceux-ci), mais aussi les personnes qui ont corésidé au moins un an et les personnes librement citées pour leur rôle clef - positif ou négatif. On dispose pour ces derniers, de leurs événements familiaux et de l'histoire de leur activité professionnelle. Dans ce questionnaire, trois générations sont décrites, l'enquêté représentant la génération pivot. À cette description de l'entourage s'ajoute le recensement de tous les événements familiaux et professionnels que l'enquêté a connus et de tous les logements que l'enquêté a occupés depuis sa naissance. Parmi les 2830 individus enquêtés, $21 \%$ ont eu plus d'une union. En retenant les unions précédentes avec enfants de l'enquêté ou de son conjoint et en tenant compte des enfants hors union, on obtient le chiffre de 628 individus ayant recomposé une famille - au moment de l'enquête ou au cours de leur trajectoire familiale - soit $22 \%$ de l'ensemble des enquêtés. Il existe cependant plusieurs manières d'appartenir à une famille recomposée 4 . L'enquêté ayant eu des enfants d'une union précédente (ou hors union) se met en couple avec un conjoint dont c'est la première union (38 \%). L'enquêté dont c'est la première union se met en couple avec une personne qui a des enfants d'une union précédente (29\%). Enfin les deux conjoints ont chacun des enfants d'une première union $(33 \%)$.

\section{Présentation des entretiens}

Dans cet article, la population a été restreinte aux personnes nées entre 1940 et 1950, de professions intermédiaires et ayant recomposé une famille à un moment donné 5 . On aurait pu supposer que ces familles recomposées de classes moyennes adopteraient un fonctionnement davantage construit sur le modèle de substitution - caractérisé par la formation d'une nouvelle famille pour effacer la précédente - que celles d'aujourd'hui, davantage formées sur le modèle de pérennité, où le maintien des relations au sein du couple parental prévaut (Théry, 1987 ; Le Gall et Martin, 1993). Néanmoins, une analyse sur ces générations permet d'intégrer le «temps long » et d'observer l'évolution du fonctionnement de ces familles depuis la séparation du couple parental, jusqu'au moment où les enfants et les beaux-enfants sont devenus adultes. Il est alors possible de restituer dans le temps les différentes logiques de

4. Dans cette recherche, c'est le point de vue des enquêtés parents qui est adopté. Par exemple, une femme divorcée seule et ayant deux enfants n'appartient pas à une famille recomposée, même si le père de ses enfants est en couple, contrairement à ses enfants.

5. Les professions intermédiaires ont été retenues dans la mesure où, même si les contraintes de budget et d'espace logement existent, elles disposent néanmoins d'une certaine marge de manœuvre dans leurs choix résidentiels et leurs attachements géographiques. 
recomposition familiale liées au logement et à l'espace, dans la mesure où elles se transforment et varient selon le contexte. Soulignons qu'il a été parfois délicat de définir une personne comme ayant recomposé une famille, ces configurations familiales étant à " géométrie variable » (Le Gall et Martin, 1988a). Dans un premier temps, nous nous sommes intéressées aux enquêtés qui avaient connu un divorce ou une séparation, ôtant ceux qui ont connu le décès de leur conjoint. Plusieurs questions permettaient de trier parmi ces personnes celles qui « reformaient » un couple. Les questions formulées permettaient également de ne pas omettre ceux qui connaissaient une union «à temps partiel » et de repérer ceux qui avaient vécu une seconde rupture. Ensuite, le questionnaire donnait des informations sur les enfants : leurs événements familiaux, leur lieu de résidence, mais aussi la fréquence des contacts avec ces derniers. Soulignons dès à présent que pour ces générations-là, les hommes indiquaient parfois ne plus avoir de contacts avec leurs enfants nés d'une première union. Pour certains, il était précisé que cette séparation s'était instaurée depuis le divorce, pour d'autres nous ne savions pas si cette rupture s'était réalisée à une date plus récente - notamment celle de l'enquête. Ces personnes n'ont pas été conservées, même si elles avaient renoué depuis une nouvelle relation et avaient des beaux-enfants, cette situation s'apparentant davantage à une logique de substitution, « sorte de veuvage social », caractéristique des premiers divorces (Théry, 1991). Néanmoins, lorsque l'enquêté était une femme et qu'elle précisait ne plus avoir de contacts avec son ex-conjoint et que celle-ci avait depuis « recomposé une famille », nous avons décidé de l'interroger. Nous étions partis de l'hypothèse qu'une rupture de relations avec l'ex-conjoint ne conduisait pas automatiquement à une absence de contacts entre le parent non-gardien et ses enfants. Effectivement, concernant les contacts avec les ex-conjoints, plusieurs questionnaires font état de contacts rompus entre ces derniers, mais les entretiens montrent que les enfants poursuivaient leurs relations avec leur père et/ou d'autres membres de la famille paternelle. Ce décalage peut notamment s'expliquer par la formulation de la question «Actuellement (ou avant son décès) qu'elle est (était) la fréquence de vos contacts ». Lorsque les enfants deviennent adultes, on peut supposer que les ex-conjoints, autrefois en relations par les questions de circulation des enfants et des décisions importantes ne poursuivent pas de contacts (Le Gall et Martin, 1988b).

Précisons enfin que le nombre limité d'entretiens ne permet pas ici de prétendre à une enquête représentative, ni de généraliser les observations. Elle offre cependant la possibilité d'explorer les familles recomposées à une autre échelle - celle d'espaces de vie - et d'ouvrir des pistes méthodologiques. Nous avons donc procédé à un recueil d'histoires et de récits de vie d'hommes et de femmes sur leur itinéraire résidentiel et dont le point commun était d'avoir vécu une recomposition familiale. Neuf femmes nées 
entre 1941 et 1950 et six hommes nés entre 1948 et 1941 ont été interrogés. Ces entretiens semi-directifs ont duré en moyenne deux heures.

\section{LES ESPACES DE CIRCULATION : LA QUESTION DE LA GARDE DES ENFANTS}

Avant d'aborder les lieux fréquentés, il semble nécessaire de retracer les relations entre ex-conjoints notamment au travers de la garde des enfants suite au divorce. Dans les générations étudiées (1940-1950), la garde alternée était une pratique rare ${ }^{6}$. Dans ces conditions, on pouvait s'attendre à l'absence de pratique de résidence alternée, avec la garde principale attribuée aux mères, choix réalisé tant par les mères que par les pères, ou plutôt devrait-on dire absence de choix, celui-ci semblant comme «allé de soi » étant données les représentations sociales de l'époque. Dans les entretiens, quelques femmes évoquent des contacts considérés comme épisodiques de leurs enfants avec leur père. Plusieurs fois, la belle-mère est apparue comme la « cause » de la relation rompue entre père et enfants, ou tout au moins d'espacement des relations. On observe néanmoins dans leur témoignage le désir de voir leur enfant poursuivre ce lien. Des actions en justice sont alors parfois intentées. Mais, plus généralement, lorsque les enquêtés sont interrogés sur la garde de leurs enfants, il leur paraît somme toute naturel que la mère ait la garde principale, tandis que le père les voit lors des vacances scolaires et des week-ends. Néanmoins, cinq entretiens font état de garde d'enfants attribuée au père. Elles sont plus souvent le résultat de situations «atypiques ». Françoise par exemple n'a pas eu la garde de sa seconde fille, née d'une seconde union. Lorsqu'elle divorce en 1985, son conjoint obtient la garde de sa fille et conserve la maison - qui était à son nom. C'est le souhait de ce dernier qui a «établi » un marché. Mais, contrairement à ce qui avait été prévu, après s'être stabilisée psychologiquement, financièrement et résidentiellement, Françoise ne reprendra pas la garde de sa fille, soulignant la complexité du système juridique. Elle la verra alors le week-end et lors des vacances scolaires. Quant à sa seconde fille, celle-ci n'a pas désiré résider chez son ex-beau-père, ni chez sa mère, préférant s'installer chez sa tante maternelle. On aura également le témoignage d'hommes, notamment d'Alain, qui demandera la garde de son fils issu de sa seconde union, arrangement établi avec son ex-conjointe (ces derniers n'étaient pas mariés). En

6. Avant 1975, année du divorce par consentement mutuel, les enfants étaient le plus souvent confiés à la garde d'un seul parent, excluant le second, et faisait référence à la faute conjugale. La pratique de la garde alternée (appelée résidence alternée aujourd'hui) s'est diffusée ensuite, mais de façon résiduelle. Celle-ci a été prohibée par l'arrêt de la Cour de cassation du 2 mai 1984. La loi Malhuret du 22 juillet 1987 a légalisé le principe de l'exercice de l'autorité parentale qui peut être commune aux deux parents divorcés, avec la désignation d'une résidence habituelle de l'enfant. Cette disposition s'est élargie en 1993 aux parents concubins. Depuis 2002, la loi du 4 mars officialise la résidence alternée. 
raison de la situation matrimoniale de son ex-conjointe - cette dernière vivait en couple avec une autre femme - il a souhaité, tout en maintenant les relations mère-fils, lui présenter une autre image du couple parental. Parfois, frères et sours n'ont pas eu le même domicile principal, les uns vivant avec leur mère, les autres avec leur père. Jacques par exemple aura la garde principale de son fils, tandis que ses filles resteront avec leur mère. Toutefois, audelà de la garde établie par la loi, on observe une véritable circulation, instaurée par les enfants, notamment lorsqu'ils sont adolescents et/ou lorsque les parents doivent gérer diverses difficultés (financières, de santé...). La décision juridique ne correspond alors pas toujours à la pratique effective. L'une des filles de Jacques le rejoindra par exemple lors de son adolescence, suite à des relations conflictuelles avec sa mère. De même lorsque Emmanuelle divorcera en 1986, elle aura la garde principale de ses deux filles. Néanmoins, on observe de fréquents allers et retours non seulement de ses filles mais également de ses belles-filles tout au long de leur adolescence. Ainsi, les enfants circulent parfois entre les domiciles des parents, pour des durées plus ou moins longues, plus ou moins définitives, ce qu'avait montré une précédente étude concernant les enfants de parents divorcés : une circulation des enfants entre les divers logements parentaux, évoluant au cours du cycle de vie et des relations parents-enfants (Clément, 2002). Des arrangements en dehors de la loi sont donc établis pour l' "intérêt de l'enfant », qui peuvent passer inaperçus dans une enquête quantitative, comme le précisait Sylvie Cadolle (2003).

\section{LES RELATIONS ET LES RENCONTRES ENTRE EX-CONJOINTS}

Si le modèle de substitution n'apparaît pas dominant dans les entretiens (tous les enfants des personnes interrogées n'ont pas rompu avec leur parent non gardien), il semble qu'un « modèle intermédiaire » puisse être délimité, où enfants et parents non-gardiens poursuivent un lien, comme dans le modèle de pérennité. Toutefois les ex-conjoints évitent les rencontres et communiquent essentiellement à travers leurs enfants. Cela peut notamment s'expliquer par l'âge des enfants lors de la rupture de leurs parents, plus souvent adolescents et donc plus à même de gérer leurs rapports avec leurs parents et la gestion de leur circulation, ce que soulignaient Didier Le Gall et Claude Martin (1988a) : l'adolescence entraînerait l'autonomisation des foyers du parent-gardien et du parent-non-gardien. Il y a bien rupture du couple conjugal d'un côté, et du couple parental de l'autre, mais maintien du lien de filiation, où l'enfant fait le lien. Parfois, les parents essaient de ne pas se rencontrer, et ce même lors des « échanges » des enfants. C'est le cas de Françoise, qui attendait dans la voiture que ses filles descendent du logement de leur père, ou d'Emmanuelle qui ne rencontre jamais son ex-conjoint pour- 
tant résidant dans la même commune qu'elle, et dont les filles étaient suffisamment âgées pour se déplacer seules. Très rares sont les relations qui se poursuivent entre les ex-conjoints en dehors des questions concernant l'éducation ou la planification des systèmes de garde. Comme le précise Jacques - qui avait la garde principale de son fils dès le divorce puis celle de l'une de ses filles lorsqu'elle eut 15 ans - des arrangements ont été réalisés afin que la fratrie se reconstitue chaque week-end et lors des vacances scolaires. Cependant les relations demeuraient «tendues » et uniquement relatives aux enfants. Les mariages des enfants et les baptêmes des petits-enfants sont alors autant d'occasions de se « retrouver », dans une ambiance qui, si elle n'est pas chaleureuse, reste « courtoise ». Et finalement, même s'ils ne se rencontrent pas, les parents ont des nouvelles respectives par le biais de leurs enfants. Des relations d'amitié donc semblent difficiles à construire. Dans les discours demeure l'importance de rester à sa place, de ne pas concurrencer les figures parentales, tant pour les parents que les beaux-parents. Lorsque les enquêtés ne signalent pas de relations rompues, ils ne cherchent pas non plus à se réunir. Si des rencontres ont lieu, elle se déroulent rarement dans le logement de l'ex-conjoint, considéré comme privé et intime. Enfin, quelques-uns ont non seulement maintenu un rapport de parentalité, mais aussi des relations d'amitié et parfois de soutien. Mais ces situations constituent davantage une exception. C'est le cas d'Évelyne par exemple qui entretient de nombreuses relations avec l'ex-belle famille de son conjoint. Toutefois, on peut se demander si ce type de fonctionnement n'est pas facilité par l'absence d'histoire familiale d'Évelyne, qui n'a jamais eu d'enfants (Blöss, 1996, 1997 ; Cadolle, 2000).

\section{LES GRANDS-PARENTS PATERNELS :}

\section{UN LIEN QUI PERDURE, UN LIEU QUI DEMEURE}

L'absence de contacts entre les ex-conjoints ne conduit pas toujours à une rupture des liens avec l'ex-belle famille. Si la majorité des enquêtés n'a plus de relations avec cette dernière, d'autres poursuivent ces liens, en particulier avec leurs ex-beaux-parents. C'est notamment le cas d'Isabelle. En effet, tout au long de son mariage, elle se rendait dans la maison de ses beauxparents, en province, accompagnée de ses enfants. Divorcée aujourd'hui de leur fils, elle n'a pas pour autant rompu les liens avec eux. Ces derniers l'invitent fréquemment chez eux, avec ses enfants. C'est alors Isabelle qui demande à ce que les liens se «distendent », leur maison étant « celle du père ». Elle désire alors « s'arracher » de cette vie affective afin d'établir une démarcation : "C'est plus mon chez moi là-bas », inscrivant alors la maison familiale à une lignée à laquelle elle n'appartient plus. Elle continuera néanmoins de leur rendre visite une à deux fois par an. Plus étonnant est l'exemple 
de Daniel, père de trois filles issues de deux mariages, et beau-père de deux enfants d'une troisième union. Originaire de Marseille, où réside toute sa famille ainsi que ses ex-conjointes, il vit actuellement à Saint-Quentin-enYvelines, avec sa troisième femme. De sa première union à l'âge de 18 ans est née une fille. Très rapidement, le couple divorce. Il n'aura plus aucun contact avec sa première femme, ni même avec sa fille. Un an après son divorce, à 21 ans, il se marie une seconde fois et a deux filles. Deux ans plus tard, il divorce de nouveau et s'installe à Bourg-la-Reine, dans l'appartement de sa troisième conjointe, qui a deux enfants. Il quitte donc Marseille mais gardera des contacts avec sa seconde femme et ses deux filles. D'après le graphe $\mathrm{n}^{\circ} 1$, on observe alors le rôle joué par Marseille dans la vie de Daniel ${ }^{7}$. En effet, les rencontres avec ses filles se déroulent chez sa mère ou chez sa sœur qui résident à Marseille, dans la même commune que ses filles et ses ex-conjointes. Fait d'autant plus marquant, les relations entre la première fille et la mère de Daniel n'ont jamais été rompues :

« Alors je me suis marié à 18 ans, à 20 ans j'ai divorcé, j'avais une fille qui a maintenant 40 piges largement, elle peut avoir 40 ans et qui est restée avec sa mère, et avec qui j'ai perdu... Sa mère voulait pas qu'elle me voit... mais tout ça ça ne se passe pas dans le drame, que j'ai retrouvée maintenant d'ailleurs et qui est une fille extraordinaire.

- Vous l'avez retrouvée à quel âge alors ?

7. Les graphiques ont été réalisés à l'aide du logiciel Réseau-lu conçu par André Mogoutov. Il s'agit de graphiques biographiques qui visent à mettre en évidence l'organisation interne des trajectoires décrites par une succession des états et des événements familiaux (naissances des enfants, mises en union, mariages, cohabitations avec les enfants et les conjoints) ou résidentiels (migrations, différents lieux de résidence, lieux fréquentés ou résidences secondaires...). Le logiciel repère dans un premier temps les états contemporains au moins une année donnée et les événements survenus au cours de l'année. Par exemple, dans le graphique 1 sont représentées en bas à droite l'installation à Bourg-la-Reine, la $3^{\mathrm{e}}$ mise en couple, la cohabitation avec les enfants de la nouvelle conjointe. À ce stade du traitement la prise en compte du temps est implicite : de la durée d'un état résulte le nombre de liens observés. Plus la durée d'un état est longue et plus la probabilité qu'il soit contemporain d'autres états de la trajectoire est forte. Le logiciel calcule pour chacun des états ou des événements un indice de centralité (nombre de liens d'un état ou d'événements/nombre de liens possibles). Tous les états ou événements sont ordonnés selon leur indice de centralité et la construction du graphique se fait de façon itérative en plaçant les états ou événements en allant du plus relié aux autres au plus isolé ; Ainsi l'absence de liens entre les états et les événements déjà placés, entraîne l'éloignement des états non contemporains (la naissance de la première fille n'a aucun lien avec l'installation à Bourgla-Reine. Les deux points se trouvent ainsi éloignés). Les points qui sont reliés aux différentes étapes de la vie matrimoniale comme Marseille et les parents se situent donc au centre du graphique. En effet, Marseille est lié aux deux premières unions puisqu'elles se déroulaient là, mais est également rattaché à la troisième union dans la mesure où Daniel y retourne régulièrement pour rencontrer sa mère et ses filles. Les cercles qui correspondent aux trois périodes conjugales ont été ajoutés de façon à permettre une lecture plus rapide. Pour plus de détails, voir l'article d'Éva Lelièvre et de Géraldine Vivier (2001). 
- Oh, ben je ne l'ai pas perdue de vue, elle n'a pas perdu de vue ma mère, sa grand-mère, mais moi non. Et puis aux 80 ans de ma mère, il y a donc trois ans elle est venue. Elle est extraordinaire, mais enfin j'ai deux autres filles qui sont aussi extraordinaires. J'ai que des filles. Donc ma deuxième femme... alors j'ai eu une première femme avec qui j'ai eu une fille, on s'est séparé, enfin on s'est séparé... on a divorcé dans la violence ! Je devais avoir 20 ans, il y a 40 ans, dans la grande violence ! n'importe quoi ! mais bon... parce que moi quand j'aimais je me mariais. Donc j'ai rencontré une fille à 21 ans, j'ai dû l'épouser, pareil, je lui ai fait deux mômes, deux filles que j'adore » (Daniel, né en 1944, technicien commercial).

Par ailleurs, ses trois filles, issues de ses deux unions se connaissent : seul Daniel ne voyait pas sa première fille. Marseille constitue alors le point commun, le nœud de toute cette famille, et c'est chez la mère de Daniel que se retrouvent les enfants, mais aussi les beaux-enfants et l'actuelle conjointe de Daniel.

«Et à Marseille vous les rencontriez chez votre mère ?

- Chez ma mère oui. Chez ma mère, chez ma sœur. Pas chez leur mère non, parce que quand même j'avais la pudeur de ne pas y aller. Non mais j'y allais tous les trois mois, donc je les prenais la journée, deux jours, donc chez ma mère, oui. Oui c'était chez ma mère.

- Donc ils avaient toujours des contacts avec leur grand-mère

- Ah oui, qu'elles ont toujours. C'est elle qui, enfin pas elle, puisque ma sœur s'occupe de ma mère, mais elles voient ma mère une fois par semaine maintenant, elles ont des enfants. Non non, ça s'est très très bien passé. Je vous dis avec la femme que j'avais, leur grand-mère c'était... mais même ma première, ma première épouse, la vache! Elle n'a jamais empêché ma première fille de voir sa grand-mère qu'elle voyait beaucoup au début quand elle était jeune, un peu moins par la suite, mais elle n'a jamais quitté ma mère. Non... voilà et ça se passait chez ma mère oui, parce que... oui voilà... ou chez ma sæur. Ma sœur a une belle maison à côté de Marseille et donc quand j'étais chez ma sœur, je prenais mes filles chez ma femme donc, on descendait même avec les enfants de ma femme, on déboulait tous là-bas, donc ils se connaissent bien. »

La grand-mère paternelle constitue ici le socle des relations familiales et c'est à travers elle et sa maison que se poursuit le lien parental. On retrouve ce qu'observait Sylvie Cadolle (2003) à propos de l'entraide dans les constellations familiales recomposées et du rôle de la grand-mère paternelle, maintenu parfois via la mère. 


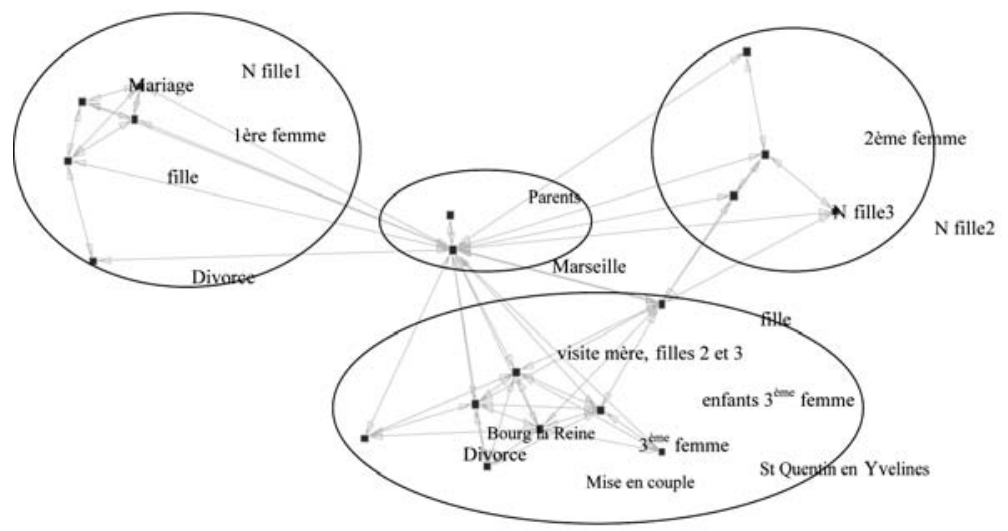

Graphe 1 - Daniel

\section{LES LIEUX FRÉQUENTÉS}

Comme le souligne Aude Poittevin (2003) dans son étude sur les fratries recomposées, « dans ce type d'ensemble fraternel, la co-résidence est centrale car elle devient l'élément commun à tous les enfants. Les noms sont différents et ne signent pas l'appartenance à la famille. Ce qui unifie n'est plus le patronyme mais l'adresse ». Agnès Martial (2003) montrait ainsi que le « vécu partagé de l'enfance » apparaissait plus essentiel que le caractère biologique, qu'il soit utérin ou consanguin. Elle précisait alors le caractère fondateur du foyer commun. Plusieurs enquêtés soulignent parfois la difficulté de « rassembler» les enfants : l'absence «d'histoires », « de culture », de « valeurs » communes, ou même «d'habitudes familiales » est souvent évoquée. C'est d'ailleurs pour l'une de ses raisons que Jeannette a préféré attendre que ses enfants quittent la maison avant de s'installer avec son conjoint.

"Mais chacun de son côté. On a terminé notre mission avant de nous mettre ensemble. Moi j'en avais trois, il en avait une. Donc il n'était pas question à ce que les enfants... alors déjà les divorces c'est dur pour... mais si encore on mélange les enfants c'est pas toujours... c'est pas toujours non plus une bonne réussite. Et pour avoir une parfaite réussite, et aujourd'hui on se rend compte, c'est ce qui se passe, c'est qu'on a peut-être eu un sacrifice disons de dix ans, un peu plus de dix ans, même plus que ça, puisqu'on a attendu que les enfants aient complètement fini d'aller à l'école, donc sachant qu'ils finissent à 25 , ça devait bien faire 15 ans, on a vécu chacun de notre côté comme ça, chacun a élevé ses enfants et aujourd'hui, bon maintenant on a des petits-enfants des deux côtés, et on a tout fait un mélange et les enfants s'entendent très très bien, 
il n'y a aucune haine, aucune rancune, tout le monde... donc on a un remerciement à ce niveau-là » (Jeannette, née en 1950, comptable).

D'après cet exemple, si l'on considère les périodes de jeunesse des enfants et des beaux-enfants, on pourrait supposer que ces derniers ont partagé peu de moments ensemble, les conjoints ayant décidé de s'installer au quotidien tardivement : leurs enfants n'ont donc pas connu une expérience de corésidence. Or, en changeant l'angle de l'analyse et en y intégrant une dimension temporelle ainsi que les différents lieux fréquentés autres que les logements paternels et maternels, on remarque le rôle des lieux de vacances et de la résidence secondaire dans la création et la construction d'un lien familial entre les différents membres de la famille. La résidence secondaire ou des lieux de vacances peuvent alors constituer un moyen de créer ce « vécu partagé ». Si l'on reprend l'exemple de Jeannette, on observe ainsi l'importance de la maison de son conjoint, située en Bretagne, où ils reçoivent leurs petits-enfants qui « reconstruisent » la famille. En effet, si leurs enfants n'ont pas été réunis sous le même toit lors de leur enfance et de leur adolescence, leur « équipe de sept petits-enfants », leur « troupe », est rassemblée dans cette résidence secondaire. Le couple envisage d'ailleurs de s'installer en Bretagne lors de la retraite de Jeannette. Concernant Alain et son épouse, ces derniers insistent sur la notion de «moments partagés » entre leurs enfants, issus d'unions différentes. Des « temps forts » à travers des « lieux points de repère » ont ainsi été agencés. Chaque année, ils allaient en vacances au même endroit, en Normandie, afin de donner à leurs enfants « des points fixes ». Quant à l'expérience de « temps fragmentés » entre frères, sœurs, demi-sœur, celle-ci a incité Jacques à créer d'autres formes de sociabilité dans divers lieux : son lieu de travail, mais aussi sa résidence secondaire et son camping car.

\section{LES LOGIQUES DE LA RÉSIDENCE SECONDAIRE}

Les familles recomposées posent la question du logement - ou des logements - le territoire des enfants se «dualisant», se multipliant. Comme le précisent Didier Le Gall et Claude Martin (1991), « la problématique du logement est interne au processus même de recomposition familiale [...] ». En s'interrogeant sur l'usage de l'espace domestique des familles recomposées, les auteurs ont montré combien l'analyse du logement - et plus particulièrement l'installation dans celui-ci, son aménagement, le partage de l'espace domestique - était révélatrice du fonctionnement de ces familles. Ils en déduisaient plusieurs logiques de recomposition et de trajectoires de logement où celui-ci « [...] participe ainsi au processus de régulation et donc de cohésion de la nouvelle famille ». Dans cette perspective, on peut s'interroger sur l'usage des résidences secondaires dans le cas des familles recomposées. 
Précisons que les familles recomposées de l'enquête «Biographies et entourage » ne possèdent pas davantage de résidence secondaire que les autres. Ainsi, $40 \%$ des couples mariés ont une résidence secondaire contre $34 \%$ des familles recomposées - au moment de l'enquête - et $21 \%$ des enquêtés qui ont recomposé une famille au cours de leur trajectoire familiale.

Prenons, l'exemple d'Isabelle, de Christophe et d'Hélène qui ont acheté une résidence secondaire au cours de leur recomposition familiale - ils sont toujours en couple au moment de l'enquête. Isabelle (graphe $\mathrm{n}^{\circ} 2$ ) et Christophe présentent de nombreuses similitudes. En effet, tous deux ont acheté une résidence secondaire avec leur actuel conjoint.

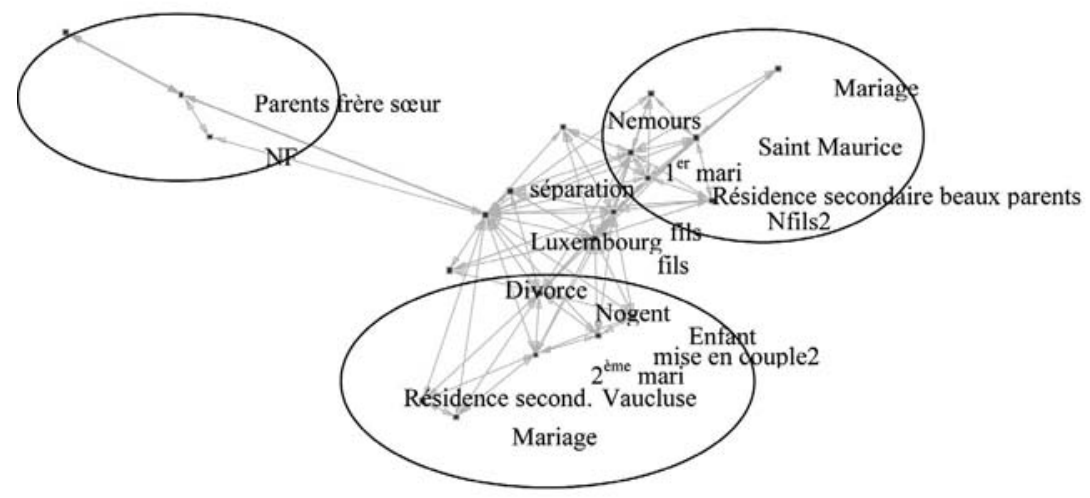

Graphe 2 - Isabelle

Isabelle a divorcé en 1982. Elle a la garde de ses enfants et conserve l'appartement qu'elle avait acheté à Nogent. Lorsqu'elle rencontre son conjoint en 1987, ils conviennent de rester dans leur appartement. Un système de circulation entre leur logement respectif est instauré ; le conjoint d'Isabelle vient plus souvent à Nogent, les enfants de celle-ci étant absents la semaine. En 1990, son conjoint, veuf, s'installe avec son fils cadet dans l'appartement d'Isabelle. En 1993, le couple achète une résidence secondaire, dans le Vaucluse. On note alors le rôle de cette maison, tant dans la trajectoire résidentielle que conjugale et familiale. En effet, la construction et l'aménagement de cette maison apparaît comme un projet conjugal, un « projet commun » que le couple a d'ailleurs concrétisé par un mariage dans cette maison.

«... on a eu envie effectivement la maison étant construite et de se marier làbas. Ça clôturait un petit peu quelque chose qu'on avait fait ensemble.

- Ça représentait un projet commun? 
- Oui. Et puis je ne dis pas qu'une maison, c'est un bébé, il faut pas... Mais je pense que c'est pas mal quand on se remarie comme ça tard d'avoir élaboré quelque chose ensemble. Il y a quelque chose qui... c'est quelque chose qui unit nos deux familles cette maison, c'est quelque chose qu'on a fait ensemble. Et c'est clair que je pense que ça renforce pas mal de choses. Ce projet commun, qui n'est pas si facile à mener, où il a fallu discuter, mettre de l'eau dans notre vin, chacun de son côté parce que qui veut une chambre comme ça, qui veut une autre. Donc c'est pas mal je trouve, je trouve c'est une chance, parce qu'on a pas tous la chance de faire ça, mais je pense que c'est pas mal. Parce qu'en se remariant à 45 ans, moi je pouvais encore avoir des enfants, le gynéco était ferme. Il m'avait dit, vraiment, vous n'êtes pas ménopausée donc... mais 45 ans, non. [...] » (Isabelle, née en 1941, infirmière).

Ici, la résidence secondaire constitue un support symbolique pour le couple : elle représente et consolide un projet commun mais elle l'inscrit également dans une continuité familiale. Cette maison réunit ainsi les deux familles : les enfants des deux lignées - celles d'Isabelle et de son conjoint ainsi que les petits-enfants, permettant ainsi de créer un sentiment d'appartenance familial à travers un lieu fixe.

« Votre maison dans le Vaucluse permet ces rencontres-là.

- Oui. Tout à fait. Ça paraît important. Ça réunit aussi les enfants de mon mari.

Il a perdu sa femme qui était d'origine grecque, et son beau-frère grec est venu passé huit jours l'été dernier. Donc cette famille là aussi peut venir chez nous. Il y a des amis de sa femme qui vont peut-être venir cet été. Donc on essaie tout de même de garder des liens dans ces familles... recomposées parce que je pense que c'est important en fait. Mais quand on a une maison effectivement dans le Vaucluse, ce n'est pas difficile de réunir la famille entre parenthèses. Une maison, je ne sais pas où dans un bled où il pleut beaucoup... »

On peut établir un parallèle avec Christophe (graphe $\mathrm{n}^{\circ} 3$ ), où l'on retrouve les mêmes termes pour décrire sa résidence secondaire. Christophe s'est marié une première fois en 1971. De cette union est née une fille en 1975. Il divorce en 1978 et se met en couple en 1981. Il aura alors deux fils avec sa seconde conjointe, tandis qu'il a la garde de sa fille les week-ends et lors des vacances scolaires. En 1987, le couple se sépare. Il emménage dans un appartement proche de son ex-conjointe afin de poursuivre les liens avec ses fils, ainsi que sa fille. Il rencontre sa troisième conjointe et s'installe en 1995 à Garches dans l'appartement de fonction de cette dernière, qui a la garde de ses deux fils. Sa fille, devenue adulte ne viendra pas dans cet appartement. Inversement, ses deux fils s'y rendent le week-end, où Christophe a d'ailleurs installé une mezzanine dans le salon afin de les accueillir. Aujourd'hui, le couple a acheté une résidence secondaire. Contrairement à Isabelle, qui hésitait à comparer une maison à un enfant, Christophe annonce 
d'emblée : «C'est notre bébé. » La résidence secondaire apparaît comme un projet conjugal, se substituant à l'enfant que le couple n'a pas eu ensemble.

« Oui, oui oui... Disons notre résidence secondaire, c'est ce qu'on a dit au début, mais on le redit de temps en temps, c'est notre bébé, parce que justement on avait notre quota d'enfants et qu'on n'a plus l'âge d'en avoir, on a investi, on s'est investi dans autre chose, donc dans cette maison, parce que les enfants, ben ils continuent à grandir, ils continuent leurs études, certains ont commencé... enfin mon grand a commencé ses études supérieures, donc eux ils sont partis pour continuer leurs études, tout ça, il était hors de question qu'on refasse un enfant tous les deux, c'était pas la peine, on a déjà assez à s'occuper avec ceux-là, donc on a investi autre chose » (Christophe, né en 1948, géomètre).

De la même façon que pour Isabelle, la résidence secondaire représente un projet commun et parachève l'union : c'est aussi dans cette résidence que le couple s'est marié en 2003. Elle est aussi le lieu de rencontre des enfants des différentes lignées, même si ce n'est pas le seul endroit où se retrouvent certains membres de la famille, notamment en Italie, chez l'actuel beau-père de Christophe. Mais dans cette résidence secondaire, les enfants de toutes les unions s'y réunissent : la fille de Christophe, née d'un premier mariage et sa petite-fille, absentes lors de ces voyages en Italie, y vont fréquemment. On remarque alors pour Christophe l'importance de se retrouver avec ses enfants.

Comme il le précise :

"Même si je vis pas avec eux, tout le reste pratiquement on peut dire que les deux tiers de leur temps disponible hors scolarité, c'est avec moi qu'ils le passent. »

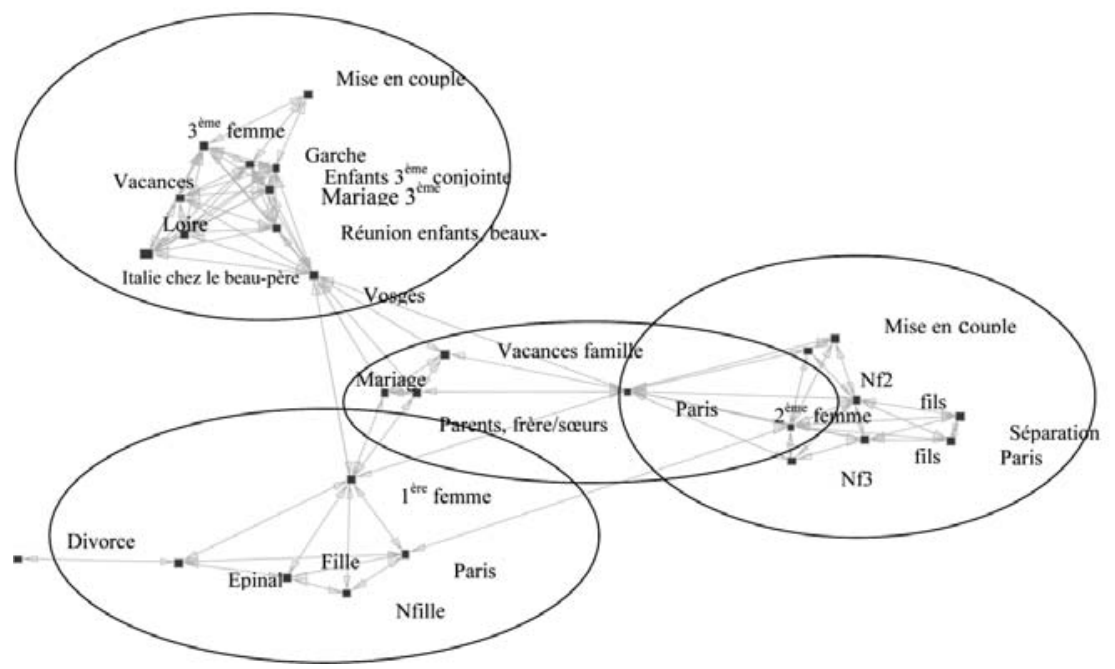

Graphe 3 - Christophe 
Si Agnès Martial (2003) précisait le caractère fondateur du foyer commun, ce n'est pas nécessairement le logement principal qui joue ce rôle comme on peut l'observer. À l'instar du logement principal, les divers lieux fréquentés peuvent aussi être un indicateur du fonctionnement familial. Ainsi, peut-on prendre l'exemple d'Hélène (graphe $\mathrm{n}^{\circ} 4$ ) qui se situe dans une logique totalement inverse. Hélène s'est mariée à l'âge de 19 ans. Elle s'installe avec son conjoint à Pontoise et aura deux filles. Après avoir acheté une maison en 1974, le couple divorce en 1977. Ils vendent la maison et Hélène, qui a la garde de ses deux filles, bénéficie d'un logement de fonction. En 1987, son second conjoint s'installe dans son appartement accompagné de son fils. Deux ans plus tard le couple se sépare. Hélène, avec sa fille cadette, se rapproche de ses parents à Béziers. Toutefois des ennuis de santé de sa fille la conduisent à retourner à Paris. C'est à cette époque qu'elle rencontre son troisième conjoint, veuf, et père de deux enfants. Pendant huit ans, ils vont garder leur logement et « circuler » entre leurs appartements. C'est lors de sa retraite qu'Hélène emménage dans la maison de son conjoint, à Chevreuse. Contrairement à Isabelle et Christophe, Hélène ne s'est pas remariée. Toutefois, on observe également pour celle-ci un système de multi-résidences : Hélène a hérité de la maison de sa grand-mère paternelle, à Bagnères-de-Bigorre, tandis qu'elle a acheté une maison dans le village où résidait sa grand-mère maternelle, à Luchon. Ce sont des lieux marquants pour Hélène : lorsqu'elle revenait du pensionnat, elle se rendait chez ses grands-mères lors des grandes vacances scolaires. Elle y est retournée fréquemment : autrefois avec son premier mari et ses enfants et avec son actuel conjoint aujourd'hui. Ils y vont ainsi chaque été. En couple, elle a acheté un appartement dans la même ville que sa fille aînée, à Carpentras. Toutefois, cet appartement n'est pas un lieu de rencontre familiale : c'est Hélène qui a entrepris les travaux, s'est occupée de l'aménagement et de la décoration. Elle s'y rend alors lorsque son conjoint et elle ont besoin de «moments seuls », son conjoint demeurant dans le « logement principal » à Chevreuse - qui n'appartient pas à Hélène (elle n'en est pas propriétaire) - forme de cohabitation intermittente observée par V. Caradec (1996) auprès des « jeunes couples âgés », mais où c'est la résidence seconde qui fait « scission » (Gotman, 1999) et permet au couple d'aménager des temps communs et des temps seuls. On a donc une logique totalement différente : l'achat de l'appartement est individuel et ne représente pas un projet commun. Il ne fixe, ni ne symbolise la famille des deux lignées : seules les filles d'Hélène s'y retrouvent. De même, les autres résidences secondaires constituent des lieux pour le couple et non pour la famille : les lignées ne se «mélangent » donc pas. Un système de multi-résidences est bien en place, mais il n'a pas la même signification ici, le lien de filiation étant non seulement préservé mais surtout distingué. Comme le précise Hélène : « Non, comme on a séparé les biens immobiliers, on a séparé aussi les familles » (Hélène, née en 1943, institutrice). 


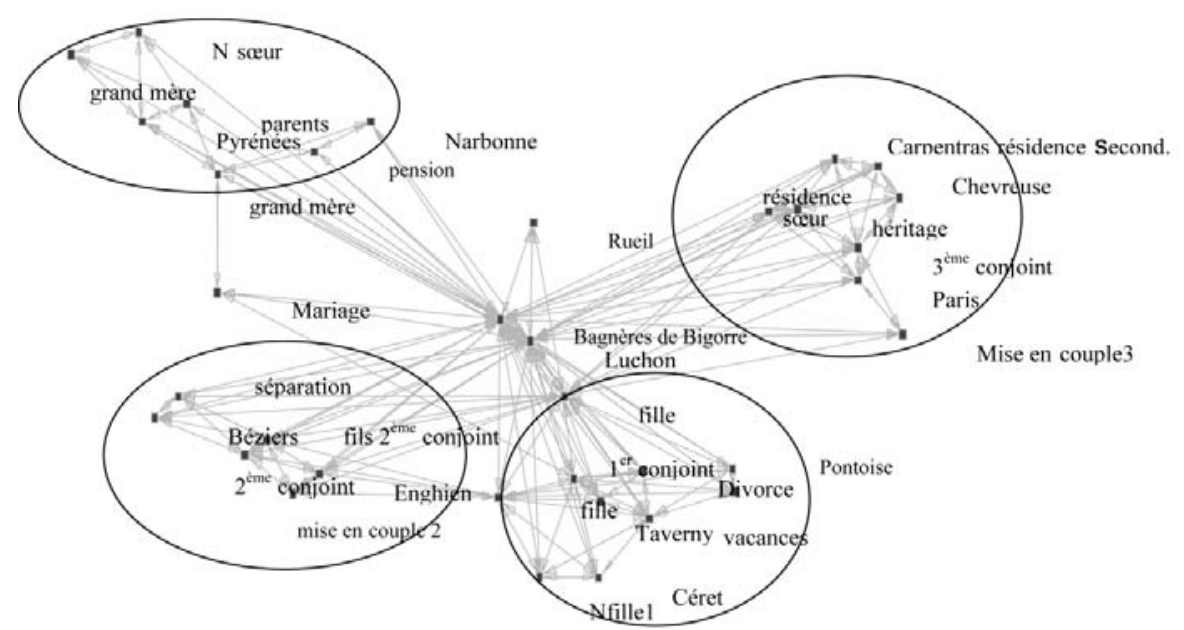

Graphe 4 - Hélène

\section{Conclusion}

Cette étude exploratoire des familles recomposées à une autre échelle - celle d'espaces de vie - a permis de souligner plusieurs points. Dans un premier temps, l'introduction du temps long et des différents lieux fréquentés a mis en évidence la complexité des trajectoires conjugales et familiales, certains des enquêtés ayant connu plusieurs recompositions familiales. Toutefois, la multiplication de ces séquences familiales ne conduit pas nécessairement à une « disparition » de certains territoires. Aussi, la famille paternelle n'est pas exclue et la grand-mère paternelle poursuit son rôle.

Cette recherche a également confirmé le rôle de la seconde résidence, particulièrement le «pôle symbolique où se recompose périodiquement la structure familiale complète » (Bonnin, 1999). Elle apparaît alors comme le centre de la vie familiale, permettant de resserrer les liens familiaux, mais aussi comme le marqueur de l'identité familiale et de son histoire. Toutefois, l'expérience du «temps fragmenté » dans les familles recomposées (Martial, 2003) semble d'autant plus favoriser la création de lieux fixes afin de reconstituer la famille. En effet, pour ces familles, il semble que « la résidence secondaire propose [...] une sorte de recentrage saisonnier à la vie de famille » (Perrot, 1998). Dans cette perspective, de la même façon que le logement principal (Le Gall et Martin, 1991), la résidence secondaire apparaît comme un indicateur du fonctionnement familial. Des logiques différentes ont ainsi été observées, où celles-ci permettaient de maintenir, de resserrer les liens de parenté, parfois même de les construire. Souvent présentée comme la maison des origines, des racines, la maison de famille - dans le cas des familles recomposées - est alors une histoire à inventer : elle 
s'inscrit dans une trajectoire conjugale et familiale dont elle constitue le point d'origine. A. Gotman (1999) relevait ainsi la dimension de continuité pour le couple de la résidence seconde, considérée « comme l'un des moyens d'accomplissement du couple et de la famille » ou moyen de « relancer le couple conjugal ». $\mathrm{Ni}$ « nouveau départ » ni « consécration »(Perrot, 1998) la seconde résidence inaugure ici l'engagement conjugal et familial. Il serait dès lors intéressant de vérifier si les résidences secondes des familles recomposées sont davantage achetées ou héritées et si le fonctionnement familial varie en fonction de ces critères. En effet, la résidence seconde n'est pas toujours destinée à inscrire le couple dans un projet commun, ni à construire de nouvelles racines de l'histoire familiale. Elle peut être au contraire le moyen de séparer non seulement les territoires conjugaux mais aussi familiaux, notamment en distinguant les logements en fonction des lignées.

\section{RÉFÉRENCES BIBLIOGRAPHIQUES}

Barbary, O. ; Dureau, F. 1993. « Des citadins en mouvement. Analyse des pratiques résidentielles à Quito (Équateur) », Cahiers des sciences humaines $n^{\circ} 29$.

BertauX-Wiame, I. 1995. "Familial et résidentiel : un couple indissociable », Sociologie et sociétés $\mathrm{n}^{\circ} 2$.

BLöss, T. 1996. Éducation familiale et beau-parenté : l'empreinte des trajectoires biographiques, Paris, L'Harmattan.

BLöss, T. 1997. Les liens de famille. Sociologie des rapports entre générations, Paris, PUF.

Bonnin, P. ; Villanova, R. de 1999. D’une maison à l'autre. Parcours et mobilités résidentielles, Paris, Grane.

Bonvalet, C. 1997. " Sociologie de la famille, sociologie du logement un lien à redéfinir », Sociétés contemporaines $\mathrm{n}^{\circ} 25$.

BonVALET, C. ; LelièVRE, É. 1995. « Du concept de ménage à celui d'entourage : une redéfinition de l'espace familial », Sociologie et sociétés $\mathrm{n}^{\circ} 2$.

Bonvalet, C. ; Maison, D. 1999. « Famille et entourage : le jeu des proximités », dans C. Bonvalet, A. Gotman et Y. Grafmeyer et al. (sous la direction de), La famille et ses proches. L'aménagement des territoires, Paris, PUF, INED, Travaux et documents, Cahier n ${ }^{\circ} 143$.

BuRguière, A. 1993. " De la famille en miettes à la famille recomposée », dans M-T. Meulders-Klein et I. Théry (sous la direction de), Les recompositions familiales aujourd'hui, Paris.

CADOLLE, S. 2000. Etre parent, être beau-parent. La recomposition de la famille, Paris, Éditions Odile Jacob.

CADOLLE, S. 2003. Entraide et solidarités dans les constellations familiales, Paris, CNAF.

CARADEC, V. 1996. «Les formes de la vie conjugale des "jeunes" couples "âgés" », Population $\mathrm{n}^{\circ} 4-5$.

CLÉMENT, C. 2002. La famille, ruptures et continuités à travers les générations, thèse de sociologie et démographie, dirigée par C. Bonvalet, Nanterre Paris X. 
Courgeau, D. 1975. «Le concept de migration », dans Migrations, état civil, recensements administratifs, actes du IV colloque de démographie africaine, Ouagadougou, 20-24 janvier 1975, Institut national de la statistique et de la démographie, p. 27-32.

Desplanques, G. 1994. «La famille : histoire d'un concept », Revue française des Affaires sociales $\mathrm{n}^{\circ} 4$.

Dubost, F. 1998. «L'autre maison. "La résidence secondaire", refuge des générations », Autrement $\mathrm{n}^{\circ} 178$.

Flandrin, J.-L. 1984. Familles. Parenté, maison, sexualité dans l'ancienne société, Paris, Édition du Seuil.

Frémont, A. ; Chevalier, J. ; Hein, R. ; Renard, J. 1984. Géographie sociale, Paris, Masson.

Gotman, A. et al. 1999. «Variations saisonnières de la vie familiale : enquête sur les secondes résidences », dans P. Bonnin, R. de Villanova (sous la direction de), D'une maison l'autre, parcours et mobilités résidentielles, Paris, Créaphis.

Le Gall, D. ; Martin, C. 1988a. Le réseau de parenté après la désunion, Paris, CNAF.

LE GALl, D. ; MARTin, C. 1998b. « Le réseau parental après un divorce ou une séparation $»$, Dialogue $\mathrm{n}^{\circ} 101$.

LE GALl, D. ; MARTIN, C. 1991. Recomposition familiale et usage de l'espace domestique, Paris, Plan Construction et Architecture.

Le Gall, D. ; Martin, C. 1993. «Transitions familiales, logiques de recomposition et modes de régulation conjugale », dans M.-T. Meulders-Klein et I. Théry (sous la direction de), Les recompositions familiales aujourd'hui, Paris, Nathan.

LE GALL, D. 1996. « Beaux-parents au quotidien et par intermittence », dans D. Le Gall et C. Martin (sous la direction de), Familles et politiques sociales. Dix questions sur le lien familial contemporain, Paris, L'Harmattan.

LELIÈVRE, E. ; VIVIER, G. 2001. «Évaluation d'une collecte à la croisée du quantitatif et du qualitatif. L'enquête Biographies et entourage », Population, 56.

Martial, A. 2003. S'apparenter. Ethnologie des liens de familles recomposées, Paris, Éditions des Sciences de l'homme.

Meulders-Klein, M.-T. ; Thery, I. (sous la direction de) 1993. Les recompositions familiales aujourd'hui, Paris, Nathan.

Pinson, D. 1988. Du logement pour tous aux maisons en tous genres, Paris, Plan Construction et Architecture, Collection « Recherches ».

Perrot, M. 1998. « La maison de famille », dans « L'autre maison. "La résidence secondaire", refuge des générations », Autrement n 178.

Poittevin, A. ; Singly F. de 2003. Les liens dans les fratries recomposées, Dossiers d'études. Allocations familiales $\mathrm{n}^{\circ} 47$.

Singly, F. de 1997. Habitat et relations familiales, Paris, Plan Construction et Architecture.

THÉRY, I. 1987. «Remariage et familles recomposées : des évidences aux incertitudes », L'année sociologique, volume 37.

THÉRY, I. 1996. (1re édition 1993), Le démariage. Justice et vie privée, Paris, Odile Jacob. 